\title{
Design of a Compact Bandstop Ulam Spiral Frequency Selective Surface
}

\author{
Rodrigo B. Moreira \\ State University of \\ Southwest Bahia \\ (UESB) \\ DCT - CPDS \\ Jequié - BA, Brazil
}

\author{
Alex F. dos Santos \\ Federal University of \\ Recôncavo da Bahia \\ (UFRB) CETENS \\ Feira de Santana - BA, \\ Brazil
}

\author{
Saulo C. Peixoto \\ State University of \\ Southwest Bahia \\ (UESB) \\ DCT - CCSI - CPDS \\ Jequié - BA, Brazil
}

\author{
Robson Maniçoba \\ State University of \\ Southwest Bahia \\ (UESB) \\ DCT - CCSI - CPDS \\ Jequié - BA, Brazil
}

\begin{abstract}
In this paper, a novel low-profile bandstop Frequency Selective Surface (FSS) is proposed for Wireless Local Area Networks (WLAN) Applications. The unit cell of the proposed FSS consists of a modification in a square patch element by the insertion of square shaped slots to obtain the Ulam spiral. The designs demonstrate a dual stopband in the WLAN frequencies bands, with angular independence.
\end{abstract}

\section{Keywords}

Frequency selective surfaces, angular stability, Ulam spiral, Dual-band, WLAN

\section{INTRODUCTION}

Frequency Selective Surface (FSS) is a kind of periodic structure and can be defined as a $2 \mathrm{D}$ or $3 \mathrm{D}$ planar array structure of identical elements on one side of the dielectric slab, widely used as spatial filters in many telecommunications applications. FSS elements can be of aperture type, patch type or an array of all-dielectric elements. These structures can exhibit bandstop or bandpass characteristics, with the proposal for blocking electromagnetic waves in certain frequencies, which depends primarily on the type and the geometry of the structure in one period called unit cell [1-5].

FSS have received special attention by several researchers over the years due to their widespread applications. The applications includes bandpass and bandstop spatial filters, absorbers, artificial electromagnetic bandgap materials, uses as superstrate of antenna or antenna array to enhance the directivity and gain [6-8]. One of the most important uses is the integration with antennas systems acting as radomes and subreflectors, the latter is applied to separate electromagnetic waves into different frequency bands.

Currently, FSS with spiral elements, angular stability, polarization independent, and multiband or wideband characteristics (transmission or reflection), has received special attention [9-16].

In this paper, a compact bandstop FSS with Ulam spiral elements providing a dual-band response, from $2.0 \mathrm{GHz}$ to $2.45 \mathrm{GHz}$ and from $4.3 \mathrm{GHz}$ to $5.8 \mathrm{GHz}$ approximately, for a $10 \mathrm{~dB}$ insertion loss reference level, is proposed. A structure of the element in the unit cell provides an angular stability and can be used for WLAN applications.

\section{ULAM SPIRAL}

Ulam spiral, also known as prime spiral, can be defined as a graphical representation of a set of prime numbers. The process consists of distributing an infinity of natural numbers in a spiral sequence that starts from the number 1 , following the sequential distribution of these numbers $(1,2,3,4, \ldots)$. The Ulam spiral is obtained by writing the positive integers numbers in a spiral arrangement. The spiral curve considered is in the counterclockwise and the position of each number in the sequence is represented by a square. Through this distribution, it is possible to highlight the squares containing the prime numbers, as can be seen in Figure 1 .

\begin{tabular}{|c|c|c|c|c|c|c|c|c|c|}
\hline 100 & 99 & 98 & 97 & 96 & 95 & 94 & 93 & 92 & 91 \\
\hline 65 & 64 & 63 & 62 & 61 & 60 & 59 & 58 & 57 & 90 \\
\hline 66 & 37 & 36 & 35 & 34 & 33 & 32 & 31 & 56 & 89 \\
\hline 67 & 38 & 17 & 16 & 15 & 14 & 13 & 30 & 55 & 88 \\
\hline 68 & 39 & 18 & 5 & 4 & 3 & 12 & 29 & 54 & 87 \\
\hline 69 & 40 & 19 & 6 & 1 & 2 & 11 & 28 & 53 & 86 \\
\hline 70 & 41 & 20 & 7 & 8 & 9 & 10 & 27 & 52 & 85 \\
\hline 71 & 42 & 21 & 22 & 23 & 24 & 25 & 26 & 51 & 84 \\
\hline 72 & 43 & 44 & 45 & 46 & 47 & 48 & 49 & 50 & 83 \\
\hline 73 & 74 & 75 & 76 & 77 & 78 & 79 & 80 & 81 & 82 \\
\hline
\end{tabular}

Fig 1: Ulam Spiral (Up to natural number 100).

The visual effect of the spiral gains more property as the number of natural numbers in the distribution increases, Figure 2. The dimensions of the squares can be of any measure, however their use is often seen with tiny sizes and the computational resources for this analysis is essential. 


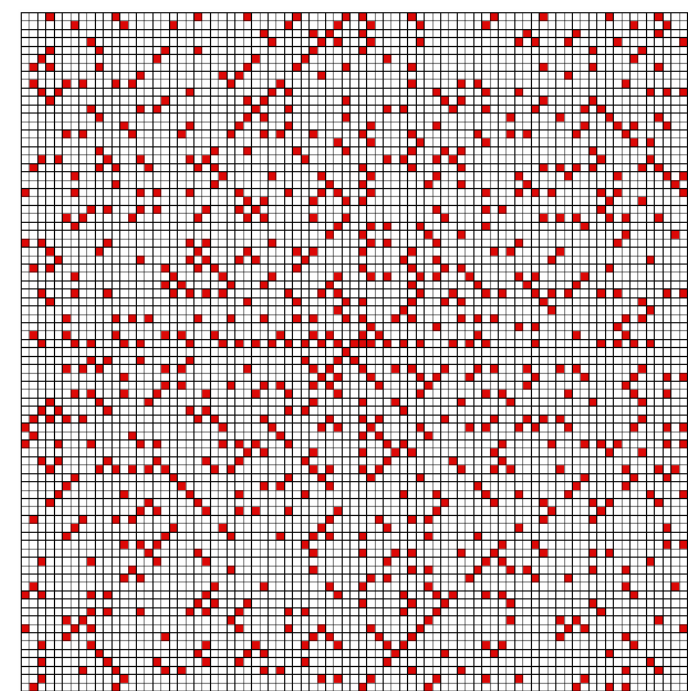

Fig 2: An 81 x 81 Ulam spiral (Source: The Ulam spiral unraveled).

\section{PROPOSED FSS UNIT CELL}

The geometry of the proposed bandstop FSS structure, from Ulam spiral, was characterized by the removal of squares with sides equal to $2.0 \mathrm{~mm}$ whose location in the distribution in the form of Ulam spiral was the location of a prime number. The proposed geometry is obtained from a square patch of dimensions $1=22.0 \mathrm{~mm}$ and $\mathrm{h}=22.0 \mathrm{~mm}$, as can be seen in the Figure 3.

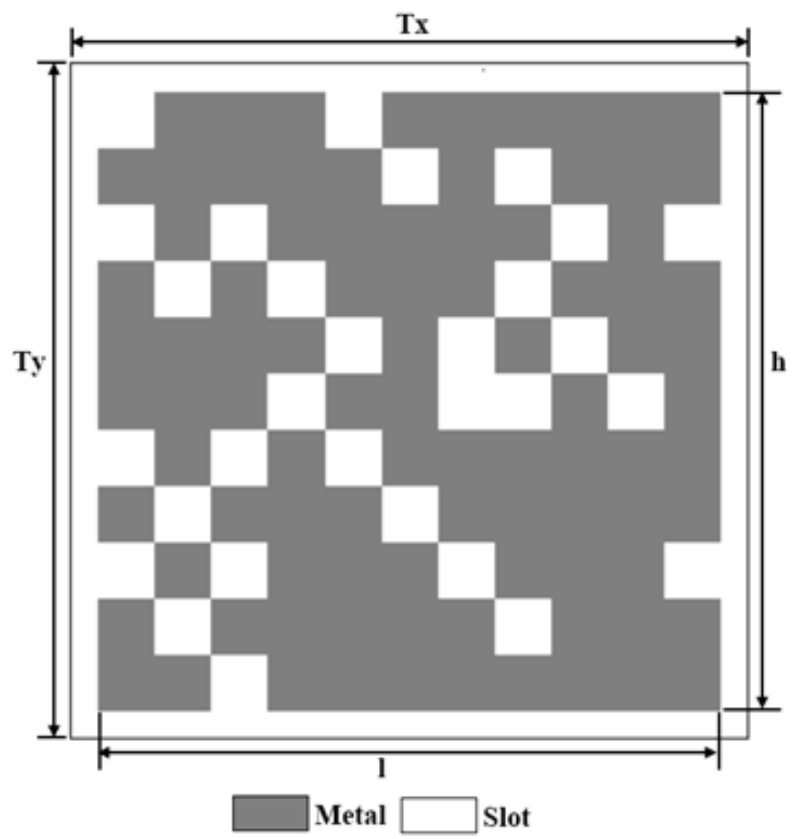

Fig 3: Unit cell of proposed FSS.

The periodicity considered for the construction of the unit cell was $\mathrm{Tx}=\mathrm{Ty}=24.0 \mathrm{~mm}$. Figure 4 represents the distribution of the numbers from 1 to 101 in the shape of Ulam spiral, as well as the emphasis given to the prime numbers that were removed from the square patch

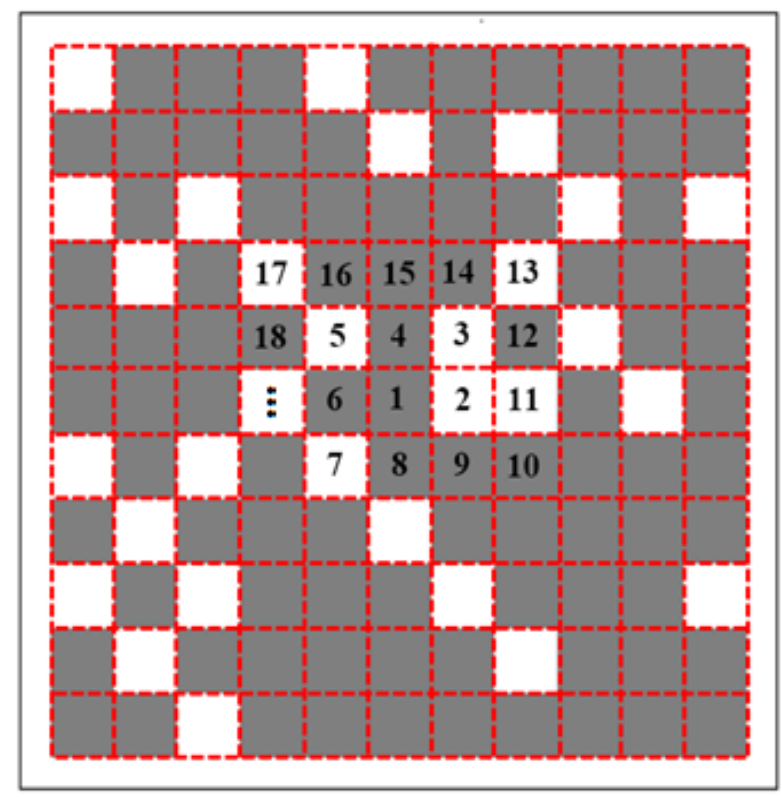

Fig 4: Representation of the spiral distribution of numbers in the FSS unit cell.

A better detail of the dimensions used in the highlighted squares, can be seen in Figure 5. It shows that the sides of the square considered were $2.0 \mathrm{~mm}$ and their locations are defined by the distribution of the numbers in a spiral shape.

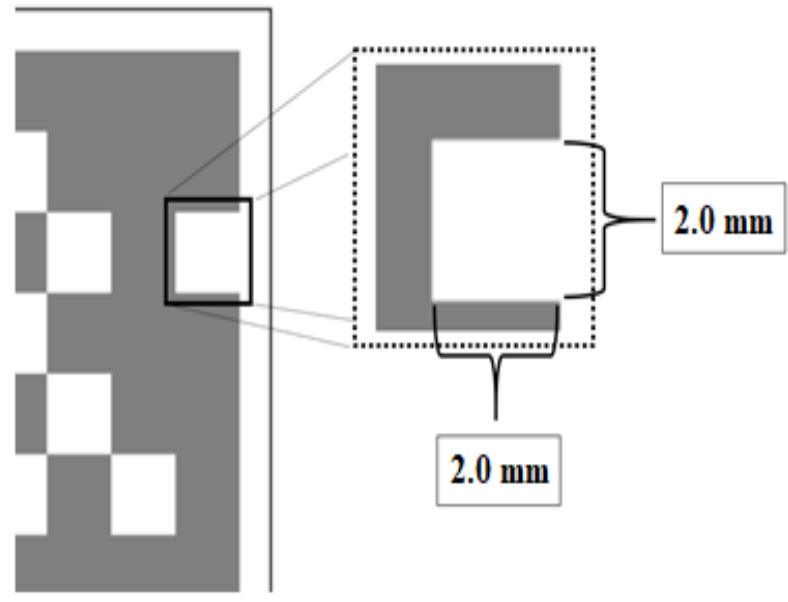

Fig 5: Dimensions of the squares for the creation of the proposed FSS unit cell.

The FSS structure is mounted on a dielectric substrate (a lowcost FR-4 substrate) with relative permittivity of 4.4 with dielectric loss tangent of 0.02 and the thickness dielectric substrate is $1.28 \mathrm{~mm}$.

\section{RESULTS}

The performance of the proposed Ulam spiral FSS in terms of transmission characteristics was obtained using Method of Moments (MoM), these results are presented in Figures 6 . Can be observed a transmission characteristics for the proposed FSS structure with resonances frequencies at 2.25 $\mathrm{GHz}(-32.83 \mathrm{~dB})$ and at $5.4 \mathrm{GHz}(-22.55 \mathrm{~dB})$, with bandwidths equal to $450 \mathrm{MHz}$ (from $2.0 \mathrm{GHz}$ to $2.45 \mathrm{GHz}$ ) and $1.50 \mathrm{GHz}$ (from $4.3 \mathrm{GHz}$ to $5.8 \mathrm{GHz}$ ), for a $10 \mathrm{~dB}$ insertion loss reference level, respectively. 


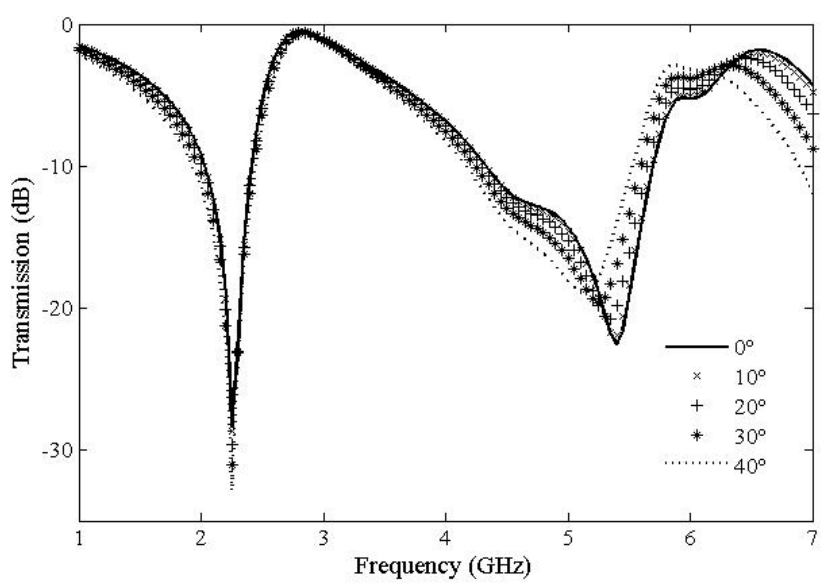

Fig 5: Transmission coefficient for the proposed FSS.

The proposed Ulam spiral FSS can be used in WLAN applications and it has very attractive characteristics, such as a low-profile structure and an angular independence. Several frequency channels used in WLAN are within the bandwidths obtained for the proposed FSS: $2.4 \mathrm{GHz}$ (IEEE $802.11 \mathrm{~b} / \mathrm{g} / \mathrm{n}$ ), and $5 \mathrm{GHz}(802.11 \mathrm{a} / \mathrm{h} / \mathrm{j} / \mathrm{n} / \mathrm{ac})$.

\section{CONCLUSION}

A compact single-layer bandstop Ulam spiral FSS is proposed in this paper. A simple modification on a traditional square patch FSS was made, squares shaped slots was inserted into it. This modification allowed to obtain a structure for WLAN applications. This FSS structure has the advantage of angular stability and dual-band frequency response at WLAN frequencies bands. As a proposal to continue this work, an investigation with the proposed structure with a different number of squares in the shape of Ulam spiral and in a multilayer format can be made to observe the behavior in terms of transmission and reflection coefficients and its bandwidths.

\section{ACKNOWLEDGMENTS}

This work is supported by Foundation for Research Support of the State of Bahia (FAPESB - Fundação de Amparo à Pesquisa do Estado da Bahia).

\section{REFERENCES}

[1] T. K. Wu, Frequency Selective Surface and Grid Array, Wiley, New York, 1995.

[2] B. A. Munk, Frequency Selective Surface: Theory and Design, Wiley, New York, 2000.

[3] A. L. P. S. Campos, A. G. d'Assunção and L. M. Mendonça, "Scattering by FSS on anisotropic substrate for TE and TM excitation", IEEE Transactions on Microwave Theory and Techniques, 50 (1), 72 - 76, 2002.

[4] J. H. Barton, C. R. Garcia, E. A. Berry, R. G. May, D. T. Gray and R. C. Rumpf, "All-Dielectric Frequency Selective Surface for High Power Microwaves", IEEE Transactions on Antennas and Propagation, 62 (7), 3652 -3656, 2014.
[5] R. Dubrovka, J. Vazquez, C. Parini and D. Moore, "Multi-frequency and multi-layer frequency selective surface analysis using modal decomposition equivalent circuit method", IET Microwaves, Antennas \& Propagation, vol. 3, pp. 492-500, April 2009.

[6] D. B. Brito, A. G. d'Assunção, R. H. C. Maniçoba and X. Begaud, "Metamaterial inspired Fabry-Pérot antenna with cascaded frequency selective surfaces", Microwave and Optical technology Letters, 55 (5), 981 - 985, 2013.

[7] H. Zhu, Y. Yu, X. Li and B. Ai, "A Wideband and High Gain Dual-Polarized Antenna Design by FrequencySelective Surface for WLAN Application”, Progress In Electromagnetics Research C (PIER C), 54, 57 - 66, 2014.

[8] D. Gangwar, S. Das, R. L. Yadava and B. K. Kanaujia, "Circularly polarized inverted stacked high gain antenna with frequency selective surface", Microwave and Optical Technology Letters, 58 (3), 732 - 740, 2016.

[9] Y. Cheng, W. Li and X. Mao, "Triple-Band Polarization Angle Independent $90^{\circ}$ Polarization Rotator Based on Fermat's Spiral Structure Planar Chiral Metamaterial", Progress in Electromagnetics Research, vol. 165, pp. 3545, 2009.

[10] K. K. Varikuntla and R. Singaravelu, "Design of a Novel 2.5D Frequency Selective Surface Element Using Fibonacci Spiral for Radome Application", in Proceedings of 2018 Asia-Pacific Microwave Conference, pp. 1289-1291, Japan, 2018.

[11] G. Yang, T Zhang, W Li and Q Wu, "A Novel Stable Miniaturized Frequency Selective Surface", IEEE Antennas and Wireless Propgation Letters, vol. 9, pp. 1018-1021, October 2010

[12] T. Liu and Sumg-S. Kim, "High-capacitive frequency selective surfaces of folded spiral conductor arrays", Microwave and Optical Technology Letters, vol. 62, pp. 301-307, January 2020.

[13] G. Yang, T Zhang, W Li and Q Wu, "A Novel Stable Miniaturized Frequency Selective Surface", IEEE Antennas and Wireless Propgation Letters, vol. 9, pp. 1018-1021, October 2010.

[14] R. Sivasamy, B. Moorthy, M. Kanagasabai, J. V. George, L. Lawrance and D. B. Rajendran, "Polarizationindependent single-layer ultra-wideband frequencyselective surface", International Journal of Microwave and Wireless Technologies, 9 (1), 93 - 97, 2017.

[15] I. Sohail, Y. Ranga, L. Matekovits, K. P. Esselle and S. G. Hay, "A Low-Profile Single-Layer UWB Polarization Stable FSS for Electromagnetic Shielding Applications", In Proceedings of the 2014 iWAT International Workshop on Antenna Technology, 220 - 223, 2014.

[16] D. B. Brito, L. M. Araújo, A. G. d'Assunção and R. H. C. Maniçoba, "A Minkowski fractal Frequency Selective Surface with high angular stability", In Proceedings of 2013 SBMO/IEEE MTT-S International Microwave and Optoelectronics Conference (IMOC), 1 - 4, 2013. 